\title{
The Scaling of QED in a Non-Commutative Space-Time
}

\author{
Jan Volkholz $* a$, Wolfgang Bietenholz ${ }^{a}$, Jun Nishimura ${ }^{b, c}$ and Yoshiaki Susaki ${ }^{b, d}$ \\ ${ }^{a}$ Institut für Physik \\ Humboldt Universität zu Berlin \\ Newtonstr. 15, D-12489 Berlin, Germany \\ ${ }^{b}$ High Energy Accelerator Research Organization (KEK) \\ 1-1 Oho, Tsukuba 305-0801, Japan \\ ${ }^{c}$ Department of Particle and Nuclear Physics \\ Graduate University for Advanced Studies (SOKENDAI) \\ 1-1 Oho, Tsukuba 305-0801, Japan \\ ${ }^{d}$ Institute of Physics, University of Tsukuba, \\ Tsukuba, Ibaraki 305-8571, Japan \\ E-mail: volkholz@physik.hu-berlin.de, \\ bietenho@physik.hu-berlin.de, \\ jnishi@post.kek.jp, susaki@post.kek.jp
}

\begin{abstract}
We present results of numerical simulations for pure $U(1)$ gauge theory in a non-commutative space. The theory is mapped onto a dimensionally reduced matrix model, which renders its numerical treatment feasible. New data on large lattices reveal the scaling of Wilson loops and their correlation functions in the simultaneous limit to the continuum and to infinite volume, at fixed non-commutativity. In this on-going project we are particularly interested in the IR behaviour, the "photo-ball" spectrum and in the photon dispersion relation.
\end{abstract}

XXIIIrd International Symposium on Lattice Field Theory

25-30 July 2005

Trinity College, Dublin, Ireland

\footnotetext{
* Speaker.
} 


\section{Non-commutative $U(1)$ gauge theory}

Somewhat overshadowed by the celebrations for Einstein's works of 1905, we are also celebrating this year the 200th anniversary of Sir William Rowan Hamilton [1], particularly here in Ireland. One of his achievements was the discovery of quaternions in 1843: he had thought for a long time about possibilities to extend the representation of complex numbers by two real components to a system with three real components, until he noticed that he had to proceed to four components to arrive at a sensible system - the first non-commutative (NC) algebra that was studied.

In the 20th century, the concept of non-commutativity for space coordinates and momenta became standard in quantum physics, but also the idea of NC space coordinates is about 60 years old [2]. It experienced a powerful renaissance in 1998, triggered by the identification of open strings at low energy with $\mathrm{NC}$ field theory [3]. NC spaces are also considered a promising approach to quantum gravity [ $\llbracket$ ]. On the phenomenological side, it might for instance explain the observation of high energy photons from far away galaxies, beyond the energy threshold apparently predicted by the Standard Model [5]. Here we study NC gauge theory in its own right.

In that framework, quantum mechanical position operators obey a commutation relation of the form $\left[\hat{x}_{\mu}, \hat{x}_{v}\right]=i \Theta_{\mu v}$, where we assume the non-commutativity tensor $\Theta$ to be constant in (Euclidean) space-time. More precisely, we consider the case of two commutative directions (which include the Euclidean time), and an NC plane with the relation $\left[\hat{x}_{i}, \hat{x}_{j}\right]=i \theta \varepsilon_{i j} \quad(i, j \in\{1,2\})$.

Field theory on such a space can be written in terms of our usual (commutative) coordinates $x$, if all field multiplications are performed by star products,

$$
\phi(x) \star \psi(x):=\phi(x) \exp \left(\frac{1}{2} \overleftarrow{\partial}_{i} \theta \varepsilon_{i j} \vec{\partial}_{j}\right) \psi(x)
$$

In particular, the action of pure $U(1)$ gauge theory takes the form

$$
S[A]=\frac{1}{4 g^{2}} \int d^{4} x \operatorname{Tr}\left[F_{\mu v} \star F_{\mu v}\right], \quad F_{\mu v}=\partial_{\mu} A_{v}-\partial_{v} A_{\mu}+i\left[A_{\mu} \star A_{v}-A_{v} \star A_{\mu}\right],
$$

which is star-gauge invariant. The Yang-Mills type self-interaction term is expected to yield a "photo-ball" spectrum [6] ${ }^{1}$. It may modify the photon dispersion relation at low energy, as it was observed non-perturbatively in the NC $\lambda \phi^{4}$ model [ [], as a consequence of UV/IR mixing effects [8]. These effects drastically complicate the perturbative treatment. However, the $\beta$ function could be computed, suggesting asymptotic freedom [9]. There are a number of further perturbative [10] and semi-classical [11] studies.

A formulation on a (fuzzy) lattice is possible [12], and it relates the spacing $a$ on a $N \times N$ lattice to the NC parameter as

$$
\theta=\frac{1}{\pi} N a^{2}
$$

Then the Double Scaling Limit (DSL), which takes simultaneously $N \rightarrow \infty$ and $a \rightarrow 0$ at $\theta=$ const. , leads to a continuous NC plane of infinite extent.

\footnotetext{
${ }^{1}$ The term "photo-ball" is an obvious analogue to the glueball; to our knowledge, it first occurred in the work by Fatollahi and Jafari quoted in Ref. [6].
} 
But such a formulation is not immediately applicable for simulations, in particular because of the request for star-unitary link variables. However, there is an exact map [13] of the NC $N \times N$ lattice onto the dimensionally reduced Twisted Eguchi-Kawai model [14] with the action

$$
S[U]=-\beta N \sum_{i \neq j} \mathscr{Z}_{i j} \operatorname{Tr}\left[U_{i} U_{j} U_{i}^{\dagger} U_{j}^{\dagger}\right], \quad \mathscr{Z}_{12}=\mathscr{Z}_{21}^{*}=\exp \{\pi i(N+1) / N\}, \quad N \text { odd }
$$

where $U_{i} \in U(N), i=1,2$. The (analogue of a) rectangular Wilson loop of sides $a I$ and $a J$ is now given by

$$
W_{i j}(I \times J)=\frac{1}{N} \mathscr{Z}_{i j}^{I J} \operatorname{Tr}\left(U_{i}^{I} U_{j}^{J} U_{i}^{\dagger I} U_{j}^{\dagger J}\right) .
$$

Mapping this quantity back to the lattice yields in fact a sensible definition of a Wilson loop in the NC gauge theory [15]. Note that NC Wilson loops are star-gauge invariant and complex.

\section{Numerical results for the Double Scaling behaviour}

This mapping of the NC plane onto a matrix model (one in each lattice site of the commutative plane) enables numerical simulations. The next challenge is to identify the dimensional lattice spacing $a(\beta)$ in order to evaluate observables in the DSL. The simple ansatz

$$
a \propto 1 / \beta
$$

turned out to be successful, as we are going to illustrate in a sequence of plots. Along with relation (1.3) it implies $N / \beta^{2}=$ const. ${ }^{2}$ We always deal with $N^{2} \times(N \pm 1)^{2}$ lattices, where $N^{2}\left((N \pm 1)^{2}\right)$ is the lattice size in the $\mathrm{NC}$ (in the commutative) plane, and the NC plane is mapped onto a twisted Eguchi-Kawai model. We present results at $N=45,55,65,71$ and 81 , and the corresponding $\beta$ values are fixed such that $N / \beta^{2} \equiv 20$ in all cases. ${ }^{3}$ This means that we are always in the weak coupling phase [14, 16].

Figure 1 (on the left) shows the real part of the Wilson loop in the NC plane as a function of the loop area. We see that the loops of the same area $I^{2} a^{2}$ reveal a convincing Double Scaling, if we insert the ansatz (2.1) (we chose the proportionality constant $=1$ ). The same is true for the phase of these Wilson loops, as the plot on the right-hand-side of Figure 1 shows. Qualitatively this behaviour is similar to the Wilson loops in 2d NC QED [17]: small loops are almost real and decay exponentially as the area increases. On the other hand, for large loops the real part oscillates around zero, and the phase grows linearly. The latter property is reminiscent of the Aharonov-Bohm effect, if one identifies $\theta$ with an inverse magnetic field across the NC plane, as suggested by Peierls [18].

Next we consider correlation functions of Wilson loops, which are located in the NC plane but separated in the (commutative) Euclidean time. As examples, we show the correlation of the real parts of $4 \times 4$ loops (on the left), and of the phases of $2 \times 2$ loops (on the right). In both cases we still observe a decent Double Scaling. Its quality improves as $N$ in increases, which indicates that our simple ansatz (2.1) does indeed describe the DSL limit asymptotically.

\footnotetext{
${ }^{2}$ In contrast, in $d=2$ we had to set $a \propto 1 / \beta^{2}$, and therefore $N / \beta=$ const. [1]].

${ }^{3} \mathrm{We}$ are also working on a systematic search for Double Scaling by matching the data without any assumption about the relation between $\beta$ and $a$. This represents a completely unbiased test of the DSL postulated here.
} 

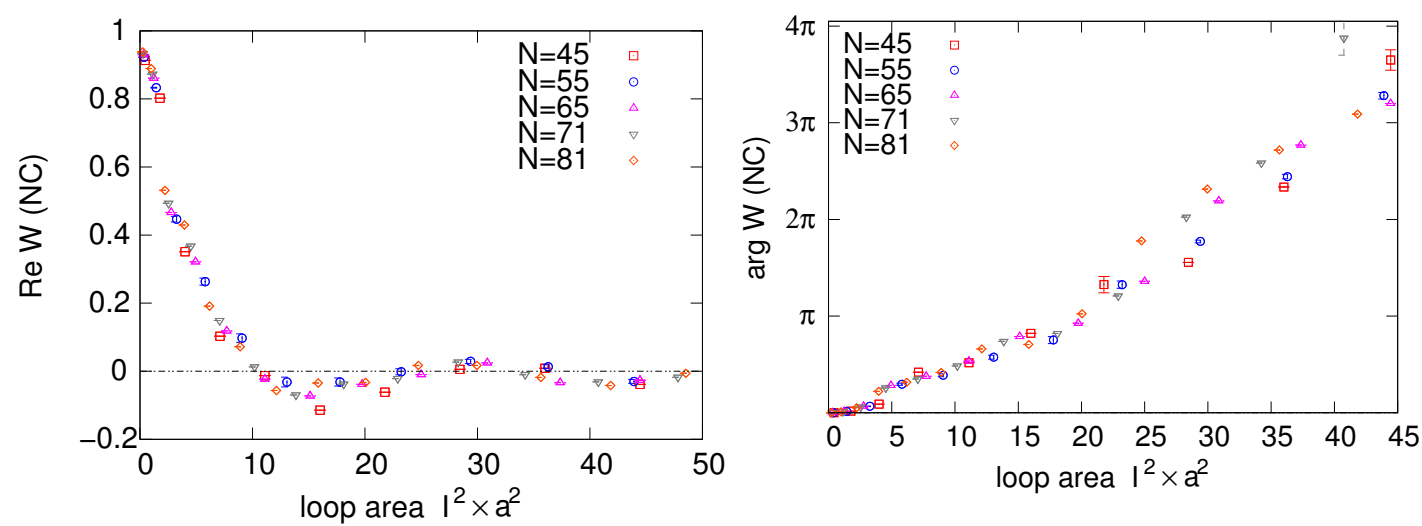

Figure 1: The Wilson loop in the NC plane: its real part (on the left) decays at small area, and for larger areas it oscillates around zero. In that regime, the complex phase (on the right) turns sizeable and it begins to grow in an (approximately) linear way.
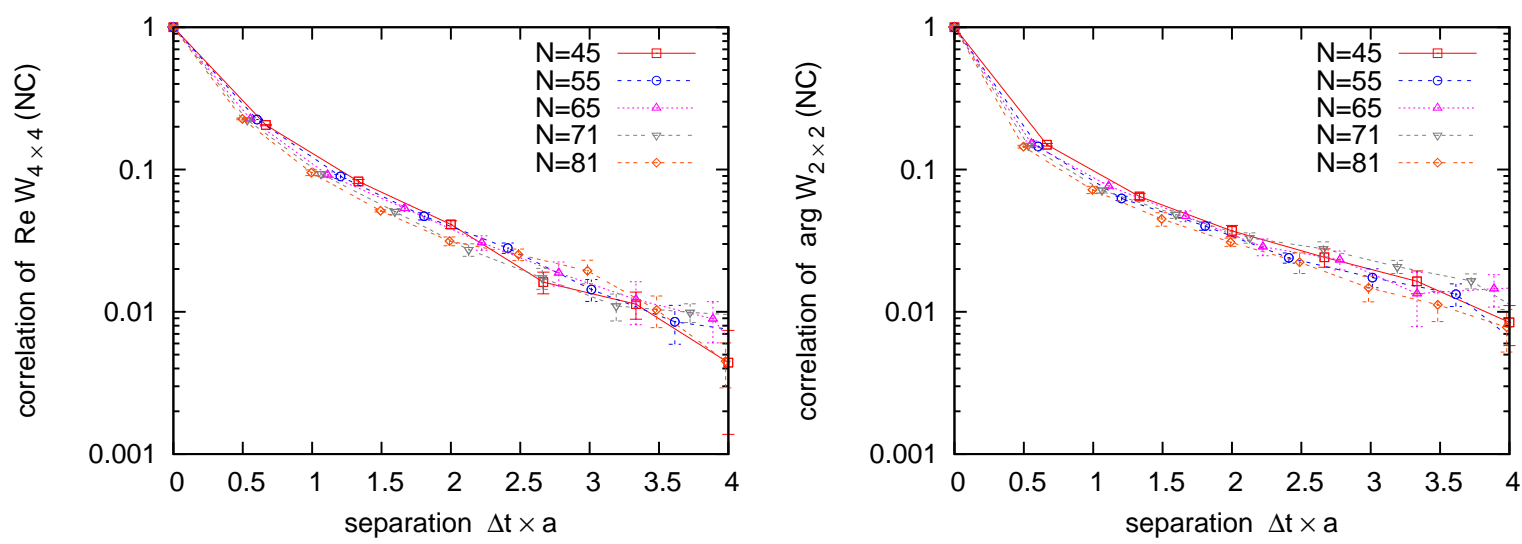

Figure 2: The correlation of Wilson loops in the NC plane, separated by a distance $a \Delta t$ in Euclidean time. Our examples are the correlation the real parts of $4 \times 4$ Wilson loops (on the left), and of the phases of $2 \times 2$ loops (on the right).

Finally we also consider Wilson loops in the commutative plane. These loops, as well as those in the mixed planes, are real due to the reflection symmetry on the commutative axes. From Figure 3 (on the left) we see that for loops in this plane the convergence towards the DSL is a little more laborious, but for our largest $N$ values it sets in also here. On the right-hand-side of this Figure we also consider the correlator of $6 \times 6$ loops in the commutative plane, again separated in the Euclidean time, which confirms the above statement with respect to the DSL.

\section{Conclusions and outlook}

We reported on our progress in a numerical investigation of pure $U(1)$ gauge theory in a NC space-time. In particular, we presented results which reveal the asymptotic rule for the Double Scaling, which takes the system to the continuum and to the infinite volume at the same time; the entanglement of these limits can be viewed in the light of the notorious UV/IR mixing. We observed that the simple ansatz (2.1) for the lattice spacing works in a satisfactory way.

Hence the bases is now provided to study the observables of physical interest and extrapolate them to the DSL. In particular, the Wilson loop correlators - examples are shown in Figure 2 

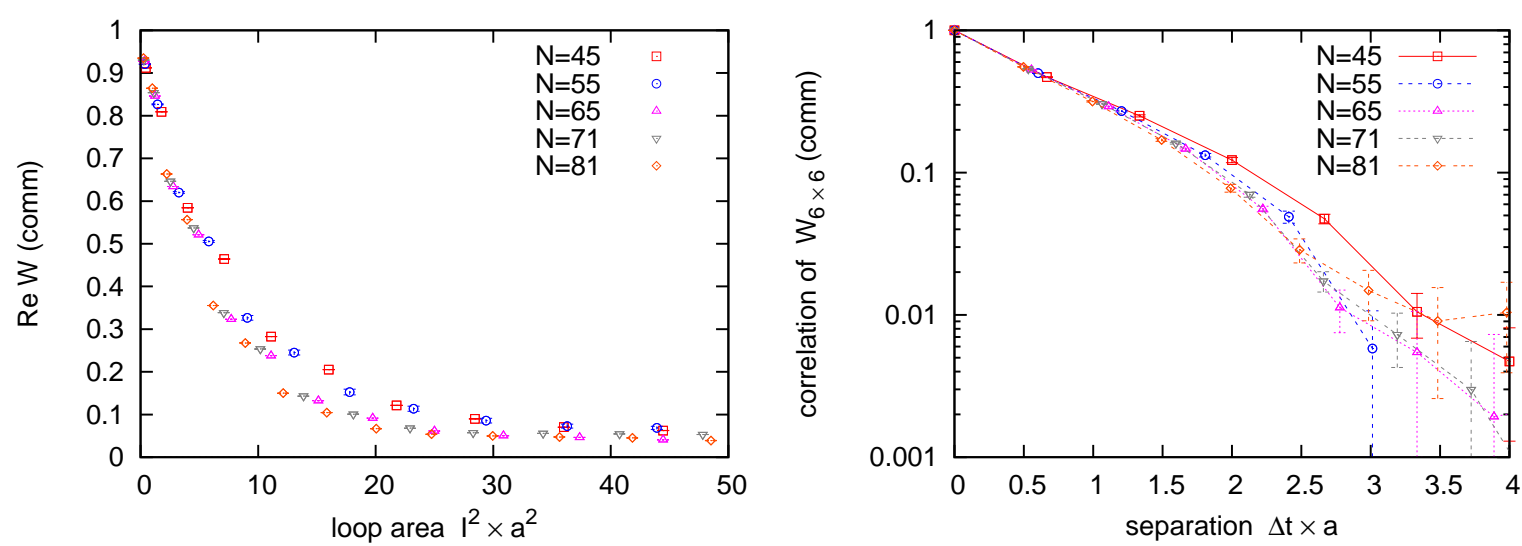

Figure 3: The Wilson loop in the commutative plane: the plot on the left depicts the loops themselves (they are real) against the dimensional area. On the right we show the correlation of $6 \times 6$ loops, separated in Euclidean time.

- provide the bases for the evaluation of the "photo-ball spectrum". We also hope to obtain results for the $\mathrm{NC}$ distortion of the photon dispersion relation, which could then be confronted with experimental data. Several high precision experiments dealing with cosmic rays are about to measure the photon dispersion to a very high accuracy, over a broad range of energies (see for instance Ref. [19]).

While our results suggest the existence of a finite continuum theory, we note that perturbative calculations revealed an infrared instability of the trivial vacuum [20] (as long as the model is not rendered supersymmetric). Indeed, we do observe numerically that the open Polyakov lines (which are star-gauge invariant as well) acquire non-zero expectation values, in accordance with perturbation theory. We therefore consider that we are actually probing a stable vacuum, which might be obtained after the condensation of "tachyons" in the trivial vacuum. This issue shall be discussed in a forth-coming paper.

Acknowledgements Frank Hofheinz has contributed to this work in an early stage, and Hinnerk Stüben has given us helpful advice regarding the parallelisation of our code. We also thank Antonio Bigarini, ChongSun Chu, Harald Grosse, Esperanza Lopez, Carmelo Perez Martin, Stam Nicolis, Denjoe O'Connor, Andrzej Sitarz, Richard Szabo and Alessandro Torielli for stimulating discussions. We are grateful for support by the "Deutsche Forschungsgemeinschaft" (DFG). Most computations were performed on the IBM p690 clusters of the "Norddeutscher Verbund für Hoch- und Höchstleistungsrechnen" (HLRN).

\section{References}

[1] L. Drury, Hamilton - the man and the mathematics, to appear in these Proc.

[2] H.S. Snyder, Phys. Rev. 71 (1947) 38. C.N. Yang, Phys. Rev. 72 (1947) 874.

[3] N. Seiberg and E. Witten, JHEP 9909 (1999) 032 [hep-th/9908142].

[4] S. Doplicher, K. Fredenhagen and J.E. Roberts, Phys. Lett. B 331 39; Commun. Math. Phys. 172 (1995) 187 [hep-th/0303037].

[5] G.T. Zatsepin and V.A. Kuzmin, JETP Lett. 4 (1966) 78. K. Greisen, Phys. Rev. Lett. 16 (1966) 748. F.W. Stecker and O.C. De Jager, Astrophys. J. 415 (1993) L71. M. Takeda et al., Astrophys. J. 522 
(1999) 225 [astro-ph/9902239]. F. Aharonian et al. (HEGRA Collaboration), astro-ph/0202072.

[6] M. Hayakawa, Phys. Lett. B 478 (2000) 394 [hep-th/9912094]. A. Matusis, L. Susskind and N. Toumbas, JHEP 12 (2000) 2 [hep-th/ 0002075 ]. F. Ruiz Ruiz, Phys. Lett. B 502 (2001) 274 [hep-th/0012171]. A.H. Fatollahi and A. Jafari, hep-th/0503078.

[7] W. Bietenholz, F. Hofheinz and J. Nishimura, JHEP 0406 (2004) 042 [hep-th/ 040402 0]. F. Hofheinz, Ph.D thesis (Berlin, 2003) [hep-th/ 0403117$]$. A. Bigarini, Ph.D. thesis, in preparation.

[8] S.S. Gubser and S.L. Sondhi, Nucl. Phys. B 605 (2001) 395 [hep-th/ 0006119 ]. G.-H. Chen and Y.-S. Wu, Nucl. Phys. B 622 (2002) 189 [hep-th/ 0110134 ]. J. Ambjørn and S. Catterall, Phys. Lett. B 549 (2002) 253 [hep-lat/ 0209106 ]. P. Castorina and D. Zappalà, Phys. Rev. D 68 (2003) 065008 [hep-th/ 0303030 ]. W. Bietenholz, F. Hofheinz and J. Nishimura, JHEP 0405 (2004) 047 [hep-th/0404179].

[9] C.P. Martin and D. Sánchez-Ruiz, Phys. Rev. Lett. 83 (1999) 476 [hep-th/9903077]. T. Krajewski and R. Wulkenhaar, Int. J. Mod. Phys. A 15 (2000) 1011 [hep-th/9903187].

[10] A. Bassetto, G. Nardelli and A. Torrielli, Nucl. Phys. B 617 (2001) 308 [hep-th/010714 7]; Phys. Rev. D 66 (2002) 085012 [hep-th/ 0205210 ]. A. Bassetto, G. De Pol and F. Vian, JHEP 0306 (2003) 051 [hep-th/0306017]. J. Ambjørn, A. Dubin and Y.M. Makeenko, JHEP 0407 (2004) 044 [hep-th/ 0406187$]$.

[11] L. Griguolo, D. Seminara and P. Valtancoli, JHEP 0112 (2001) 024 [hep-th/ 0110293 ]. L.D. Paniak and R.J. Szabo, Commun. Math. Phys. 243 (2003) 343 [hep-th/ 0203166 ]; JHEP 0305 (2003) 029 [hep-th/ 0302162 ]. A. Bassetto and F. Vian, JHEP 0210 (2002) 004 [hep-th/ 0207222 ]. H. Dorn and A. Torrielli, JHEP 0401 (2004) 026 [hep-th/0312047].

[12] For a review, see R.J. Szabo, Phys. Rep. 378 (2003) 207 [hep-th/ 0109162 ].

[13] H. Aoki, N. Ishibashi, S. Iso, H. Kawai, Y. Kitazawa and T. Tada, Nucl. Phys. B 565 (2000) 176. [hep-th/9908141]. J. Ambjørn, Y.M. Makeenko, J. Nishimura and R.J. Szabo, JHEP 9911 (1999) 29 [hep-th/ 9911041 ]; Phys. Lett. B 480 (2000) 399 [hep-th/ 0002158 ]; JHEP 0005 (2000) $023[$ hep-th/0004147].

[14] A. González-Arroyo and M. Okawa, Phys. Rev. 27 D (1983) 2397.

[15] N. Ishibashi, S. Iso, H. Kawai and Y. Kitazawa, Nucl. Phys. B 573 (2000) 573 [hep-th/9910004]. D.J. Gross, A. Hashimoto and N. Itzhaki, Adv. Theor. Math. Phys. 4 (2000) 893 [hep-th/ 0008075$]$. A. Dhar and Y. Kitazawa, Nucl. Phys. B 613 (2001) 105 [hep-th/0104021].

[16] W. Bietenholz, F. Hofheinz, J. Nishimura, Y. Susaki and J. Volkholz, Nucl. Phys. B (Proc. Suppl.) 140 (2005) 772 [hep-lat/ 040905 9]. W. Bietenholz, A. Bigarini, F. Hofheinz, J. Nishimura, Y. Susaki and J. Volkholz, Fortschr. Phys. 53 (2005) 418 [hep-th/0501147].

[17] W. Bietenholz, F. Hofheinz and J. Nishimura, JHEP 0209 (2002) 9 [hep-th/ 0203151$].$

[18] R. Peierls, Z. Phys. 80 (1933) 763.

[19] L. Latronico, Nucl. Instrum. Meth. A 511 (2003) 68.

[20] K. Landsteiner, E. Lopez and M.H. Tytgat, JHEP 0106 (2001) 055 [hep-th/ 0104133 ]. M. van Raamsdonk, JHEP 0111 (2001) 006 [hep-th/ 01100 93]. A. Armoni and E. Lopez, Nucl. Phys. B 632 (2002) 240 [hep-th/ 0110113 ]. Z. Guralnik, R.C. Helling, K. Landsteiner and E. Lopez, JHEP 0205 (2002) 025 [hep-th/ 0204037 ]. 